\title{
Internal tides and energy fluxes over Great Meteor Seamount
}

\author{
T. Gerkema and H. van Haren \\ Royal NIOZ, P.O. Box 59, 1790 AB Den Burg, Texel, The Netherlands \\ Received: 1 March 2007 - Published in Ocean Sci. Discuss.: 3 April 2007 \\ Revised: 20 September 2007 - Accepted: 24 September 2007 - Published: 1 October 2007
}

\begin{abstract}
Internal-tide energy fluxes are determined halfway over the southern slope of Great Meteor Seamount (Canary Basin), using data from combined CTD/LADCP yoyoing, covering the whole water column. The strongest signal is semi-diurnal and is concentrated in the upper few hundred meters of the water column. An indeterminacy in energy flux profiles is discussed; it is argued that a commonly applied condition used to determine these profiles is in fact invalid over sloping bottoms. However, the vertically integrated flux can be established unambiguously; the observed results are compared with the outcome of a numerical internal-tide generation model. For the semi-diurnal internal tide, the vertically integrated flux found in the model corresponds well to the observed one. The observed diurnal signal appears to be largely of non-tidal origin.
\end{abstract}

\section{Introduction}

Recent estimates, based on satellite altimetry and modelling, indicate that barotropic tides lose about one third of their energy in the deep ocean (Egbert and Ray, 2003); this loss occurs predominantly over rough topography. From these findings, supplemented by in-situ observations, one can infer that the principal process responsible for this loss is internal-tide generation, a process in which energy is transferred from barotropic to baroclinic tides. Observations at the Hawaiian Ridge support this idea; internal-tide energy fluxes of the order of $10 \mathrm{~kW} \mathrm{~m}^{-1}$ were found at various locations (Rainville and Pinkel, 2006; Nash et al., 2006), and the total loss of barotropic tidal energy, for all the tidal constituents together, in the near-Hawaiian area is estimated at nearly $25 \mathrm{GW}$ (Zaron and Egbert, 2006). Of this amount, an estimated $15 \%$ is lost to turbulence in the vicinity of the ridge,

Correspondence to: T. Gerkema

(gerk@nioz.nl) presumably by cascading of internal-tide energy to smaller scales (Klymak et al., 2006).

The basic definition of internal-tide energy flux is $E_{f}=\left\langle u^{\prime} p^{\prime}\right\rangle$, where brackets denote the time-average over a tidal period; $u^{\prime}$ and $p^{\prime}$ are the baroclinic velocity component (in the direction of the energy flux) and baroclinic pressure, respectively. Since baroclinic pressure cannot be measured directly, one has to resort to indirect methods, using for example isopycnal excursions. From this, baroclinic pressure can be derived, save for a constant of integration. Attempts have been made to determine this constant. Kunze et al. (2002) proposed a "baroclinicity condition for pressure" to the effect that its vertical integral is assumed to be zero; this indeed fixes the constant. Although they added a cautionary remark ("this condition may not hold in regions of direct forcing"), they did not restrain its application to regions away from topography, nor did later authors (Nash et al., $2005,2006)$. So, it has been indiscriminately applied over large canyons and ridges, even though its validity has not been demonstrated. We show here that the condition is in fact invalid over topography because it is incompatible with the other baroclinicity condition, that for horizontal velocity (see Sect. 4.1). We argue that it is fundamentally impossible to find the constant from single-profile measurements, implying an unresolvable indeterminacy in the energy flux profiles. However, the constant is immaterial to the vertically integrated energy flux, so this quantity can be determined unambiguously.

The main purpose of this paper is to present observations over Great Meteor Seamount and to derive the vertically integrated internal-tide energy fluxes. Great Meteor Seamount lies in the western part of the Canary Basin, halfway between the Canary Islands and the Mid-Atlantic Ridge. It is a guyot, named after the research vessel "Meteor" with which it was discovered in 1938 (Dietrich, 1970). In recent years, the currents, tidal or otherwise, and stratification around Great Meteor Seamount have been studied; van

Published by Copernicus Publications on behalf of the European Geosciences Union. 


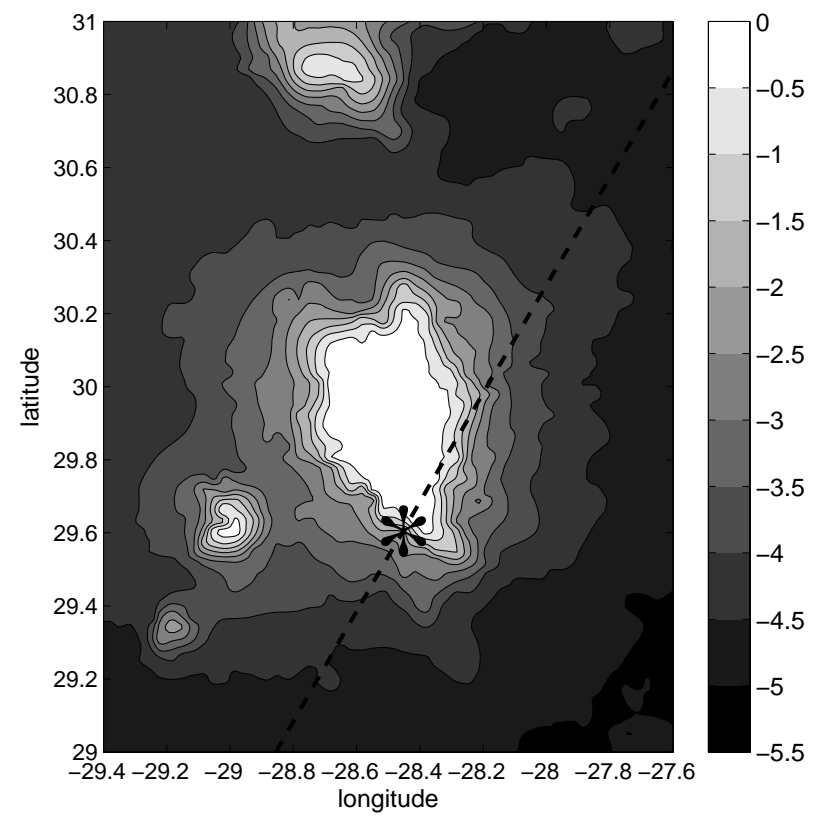

Fig. 1. Great Meteor Seamount, with the location of the CTD/LADCP yoyo-station at the center of the asterisk $\left(29.61^{\circ} \mathrm{N}\right.$, $28.45^{\circ} \mathrm{W}$ ), and the track used in the numerical calculations indicated by the dashed diagonal. Depth is in $\mathrm{km}$. This map was constructed from the database by Smith and Sandwell (1997). The top of the seamount is formed by a large plateau, where depths lie typically between 300 and $500 \mathrm{~m}$.

Haren (2005a) found a time-variability of the bottom boundary layer over this seamount. In the course of minutes, a steep front or bore may pass, whose overturning diminishes the local stratification profoundly; during the remainder of the tidal period the stratification is gradually reconstituted. An overview of the hydrography around Great Meteor Seamount was given by Mohn and Beckmann (2002), based on observational and modelling work. Besides a near southwestward flow, being part of the wind-driven subtropical gyre, they found semi-diurnal and diurnal barotropic and baroclinic tides (we discuss some of their specifics below). As Great Meteor Seamount covers, approximately, the latitudinal range $29.5-30.5^{\circ} \mathrm{N}$, diurnal components $\mathrm{K}_{1}$ and $\mathrm{O}_{1}$ are locally near-inertial.

The measurements presented here were made by simultaneous CTD and LADCP (Lowered Acoustic Doppler Current Profiler) yoyoing over the slope of Great Meteor Seamount, during $241 / 2 \mathrm{~h}$. The data are presented in Sect. 2. A harmonic analysis is applied to extract the semi-diurnal and diurnal components (Sect. 3). From this we derive the vertically integrated energy fluxes of the semi-diurnal and diurnal internal tides (Sect. 4.2). A comparison with a numerical internal-tide generation model is made in Sect. 5 .
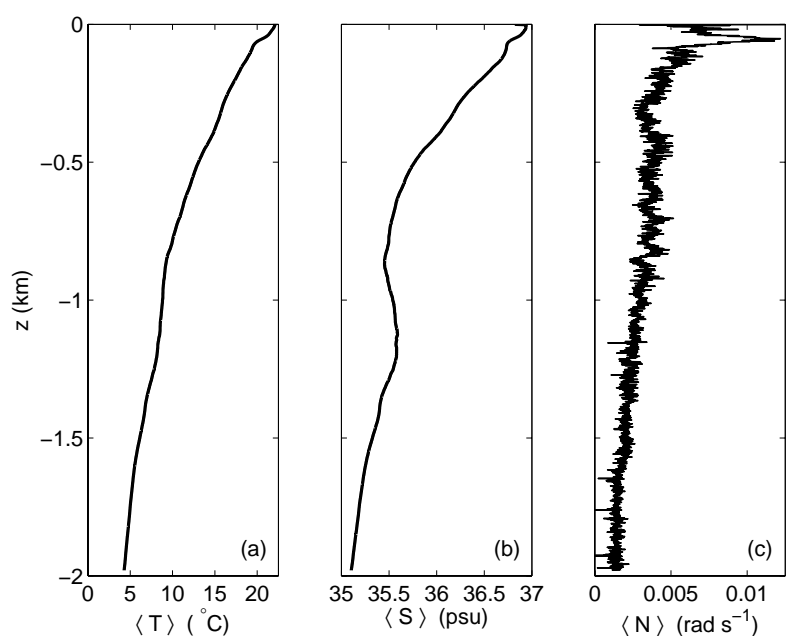

Fig. 2. Time-averaged profiles of temperature, salinity, and buoyancy frequency, derived from the full set of CTD yoyo-casts.

\section{Measurements}

The area of investigation is Great Meteor Seamount, centered around $30^{\circ} \mathrm{N}, 28.5^{\circ} \mathrm{W}$. Combined CTD/LADCP yoyoing was carried out approximately halfway up its southeastern slope, at the spot marked in Fig. 1, where the water depth is $1980 \mathrm{~m}$. The measurements started at $08: 45$ UTC on 7 June 2006, and continued until 09:15 UTC the next day (van Haren, 2006); in the figures shown below, we refer to the start as $t=0$. In this timespan of $24.5 \mathrm{~h}, 20$ casts were made.

The instrumental package was lowered and hoisted between $5 \mathrm{~m}$ from the surface and the bottom at a speed of about $1 \mathrm{~m} \mathrm{~s}^{-1}$. The package consisted of a Sea-Bird 911plus CTD sampling at $24 \mathrm{~Hz}$. For the present purposes, the CTD data were vertically subsampled at intervals of $0.5 \mathrm{dbar}$. On the same frame, two $300 \mathrm{kHz}$ RDI ADCPs were mounted, one upward looking, the other, downward; together they form the LADCP. The ADCPs sampled currents at depth intervals between $8-20 \mathrm{~m}$ from their head at an accuracy of about $0.05 \mathrm{~m} \mathrm{~s}^{-1}$.

\subsection{Temperature and salinity}

In the analysis of the temperature and salinity data, up- and down casts of the CTD were treated separately, making the total number of vertical profiles twice that of the number of casts. The data were interpolated to a regular time-grid with steps of half an hour, and vertically interpolated to a grid with $\Delta z=0.5 \mathrm{~m}$. The time-averaged signal is shown in Figs. $2 \mathrm{a}, \mathrm{b}$. A conspicuous feature is the local salinity maximum at about $1100 \mathrm{~m}$ depth (accompanied by a less noticeable increase in temperature), which is due to the outflow of Mediterranean water. 


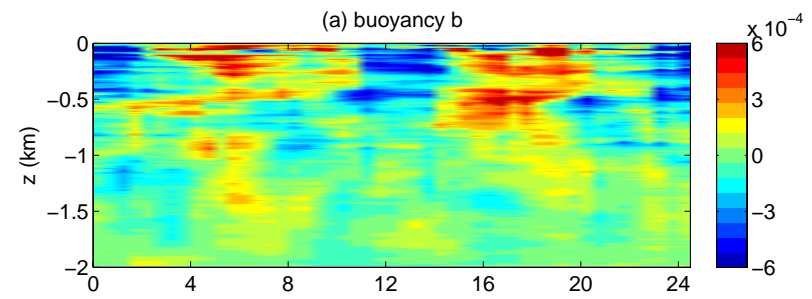

(b) isopycnal excursion $\zeta$

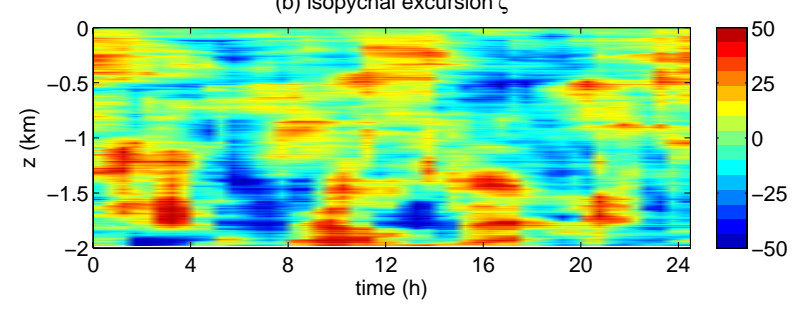

Fig. 3. Results derived from the CTD yoyo-casts: buoyancy $b$ (in $\mathrm{m} \mathrm{s}^{-2}$ ), as defined in Eq. (1), and the isopycnal excursion $\zeta=-b /\left\langle N^{2}\right\rangle$ (in $\left.\mathrm{m}\right)$

The buoyancy frequency $N$ can be determined using its basic definition

$N^{2}=g^{2}\left(\frac{d \rho}{d p}-\frac{1}{c_{s}^{2}}\right)$.

Here $\rho$ is the in-situ density and $c_{s}$ the speed of sound; these quantities were calculated as functions of pressure, temperature and salinity using the equation of state for the Gibbs potential (Feistel and Hagen, 1995). The derivative $d \rho / d p$ was approximated by discretization with steps $\Delta p$ of $0.5 \mathrm{dbar}$. The time-averaged profile of $N$ is shown in Fig. 2c. In a few instances, $N^{2}$ is slightly negative; they are here rendered by $N=0$.

Having obtained the in-situ density $\rho$ from the equation of state, we can calculate its time-averaged value $\langle\rho\rangle$, and hence buoyancy $b$ defined by

$b=-g \frac{\rho-\langle\rho\rangle}{\rho_{*}}$,

where $\rho_{*}$ is the mean of $\langle\rho\rangle$ over the vertical. So, $b$ represents the departure of density from its time-average, scaled by a factor $-g / \rho_{*}$. The field $b$, as a function of vertical and time, is shown in Fig. 3a. The predominantly semi-diurnal character of the signal is obvious, especially in the upper part of the water column. Vertical isopycnal displacements $\zeta$ can be derived from $b$ via $\zeta=-b /\left\langle N^{2}\right\rangle$, see Fig. 3b. Peak amplitudes as large as $75 \mathrm{~m}$ are reached at some points (for clearer representation, the amplitude-range is however restricted to $50 \mathrm{~m}$ in Fig. 3b). The stripiness of the signal through the vertical is due to small-scale variations in $\langle N\rangle$, cf. Fig. 2c. In the deeper parts of the water column, a weak quarter-diurnal signal is visible.
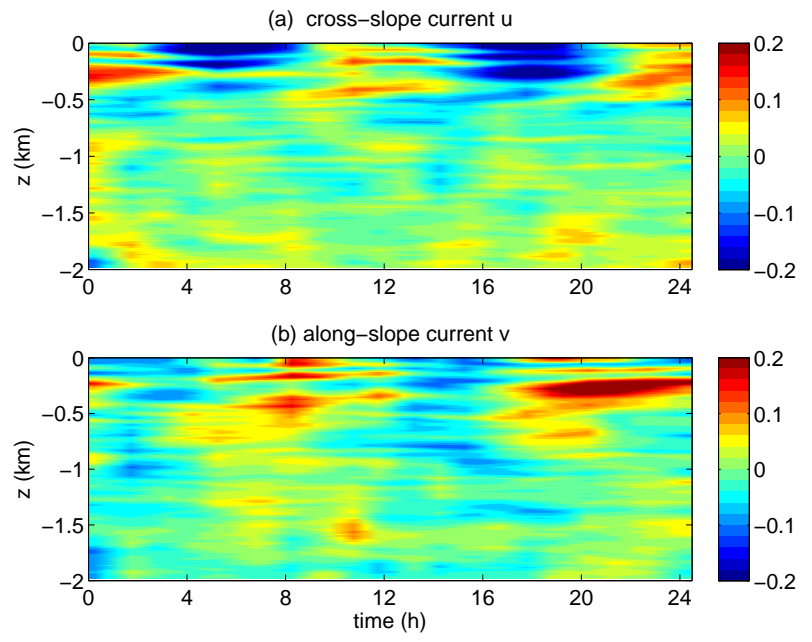

Fig. 4. Results from the LADCP yoyo-casts: the total cross-slope and along-slope velocity components, in $\mathrm{m} \mathrm{s}^{-1}$.

\subsection{Currents}

In the LADCP measurements the up- and downcasts were combined in the postprocessing to correct for systematic errors; hence the records provide 20 vertical profiles from the casts. The original set contains data every $20 \mathrm{~m}$ in the vertical, which we interpolated to a grid of $\Delta z=0.5 \mathrm{~m}$ for consistency with the CTD data and later handling. The horizontal velocity was decomposed into a cross-slope component $u$, taken along the dashed diagonal in Fig. 1 (positive in the northeastern direction), and, perpendicularly to it, an along-slope component $v$ (positive in the northwestern direction). Figure 4 shows the full signals $u$ and $v$; the predominantly semi-diurnal character is clearly visible. A shift to offslope currents is visible in the upper $400 \mathrm{~m}$ in Fig. $4 \mathrm{a}$ (blue dominates), indicative of a southwestern background current, which fits in with the overall pattern of the eastern branch of the subtropical gyre (Mohn and Beckmann, 2002). Also, one finds in Fig. $4 \mathrm{~b}$ that northwestern currents slightly dominate around $300 \mathrm{~m}$ (red dominates); these features, indicative of time-mean currents, are further illustrated in Fig. 5.

\section{Harmonic analysis of observed records}

The time-span of the data presented in the previous section $(24.5 \mathrm{~h})$ is obviously too short to resolve distinct semi-diurnal constituents such as the lunar component $\mathrm{M}_{2}$ and the solar $\mathrm{S}_{2}$, let alone various diurnal constituents such as $\mathrm{K}_{1}, \mathrm{O}_{1}$, and the inertial period. In the following analysis, we therefore lump nearby constituents together, and distinguish only the categories "diurnal", "semi-diurnal", "quarter-diurnal", and a "time-mean". 


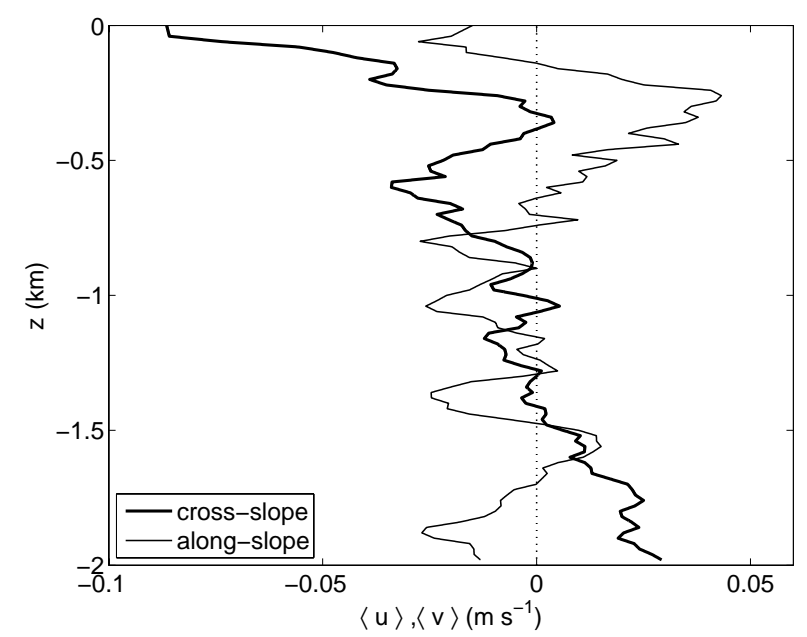

Fig. 5. Vertical profiles of the cross- and along-slope time-mean currents.

Let the original field $q_{o r}$ (standing for current components, buoyancy etc.) be approximated by the superposition

$q=\sum_{n} a_{n} \sin \left(\sigma_{n} t-\phi_{n}\right)$,

where $\sigma_{n}$ are the frequencies $\sigma_{0}=0$ (time-mean), $\sigma_{1}=7.292 \times 10^{-5} \quad\left(\mathrm{~K}_{1}\right.$, diurnal $), \sigma_{2}=1.405 \times 10^{-4} \quad\left(\mathrm{M}_{2}\right.$, semi-diurnal), and $\sigma_{3}=2 \times \sigma_{2}\left(\mathrm{M}_{4}\right.$, quarter-diurnal $)$, all in $\operatorname{rad~s}^{-1}$. The amplitudes $a_{n}$ and phases $\phi_{n}$ are given by

$$
\begin{aligned}
& a_{n}=2\left(\left\langle q \sin \sigma_{n} t\right\rangle^{2}+\left\langle q \cos \sigma_{n} t\right\rangle^{2}\right)^{1 / 2} ; \\
& \tan \phi_{n}=-\left\langle q \cos \sigma_{n} t\right\rangle /\left\langle q \sin \sigma_{n} t\right\rangle,
\end{aligned}
$$

where $\langle\cdot\rangle$ stands, as before, for time-averaging over the whole record. In this procedure, we treat different constituents as if they were orthogonal, mimicking a Fourier decomposition. The validity of this procedure can be checked a posteriori by comparing the original signal $q_{\text {or }}$ with the sum (2); we carried out such checks and found that the two were always very similar (an example is shown in Fig. 6).

We present the results of this decomposition for the crossslope and along-slope currents. The time-mean flow is shown in Fig. 5; it confirms the presence of a flow that is predominantly directed off the seamount in the upper layer, as noted above already. We split the time-dependent constituents (i.e., diurnal, semi-diurnal and quarter-diurnal) of the velocity fields into two parts: a depth-averaged, or barotropic part, and the remainder, or baroclinic part. The barotropic crossslope flow is shown in Fig. 6. Amplitudes are: 0.02 (semidiurnal), 0.0075 (diurnal), and 0.0024 (quarter-diurnal), all in $\mathrm{m} \mathrm{s}^{-1}$. The semi-diurnal constituent is 2.7 times stronger than the diurnal one. This factor falls within the range of values observed by Mohn and Beckmann (2002), who found the following typical values for the tidal/inertial constituents (all

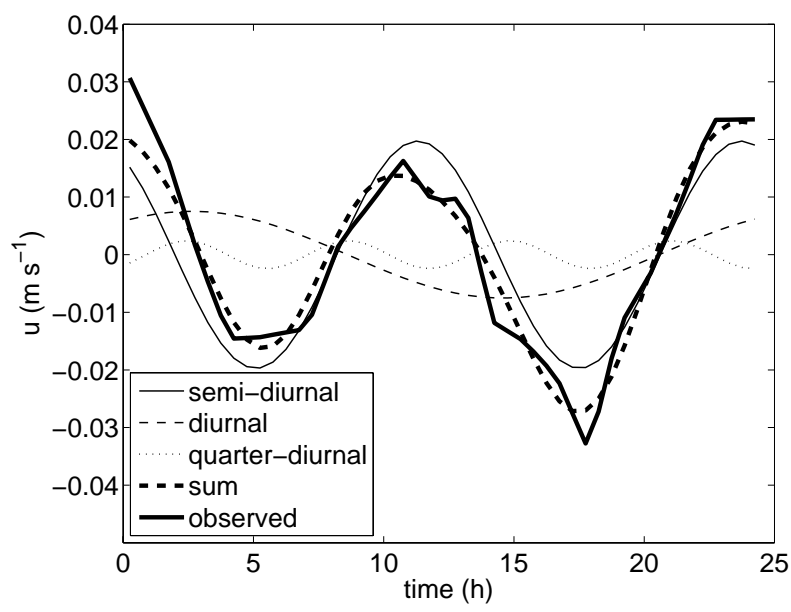

Fig. 6. The harmonic constituents, and their superposition, of the cross-slope barotropic flow. An indication of the accuracy of the fit is given by \langle| sum-observed |\rangle$/\langle|$ observed |\rangle$=0.19$, i.e. the fit deviates on average by $19 \%$.

in $\mathrm{m} \mathrm{s}^{-1}$ ): $\mathrm{M}_{2}, 0.14 ; \mathrm{S}_{2}, 0.04 ; \mathrm{K}_{1} / \mathrm{f}, 0.03 ; \mathrm{O}_{1}, 0.02$. The diurnal components together thus are 2 to 3.6 times smaller than the semi-diurnal ones, depending on the moment within the spring-neap cycle. Our measurements were made approximately half-way between first-quarter and full moon, so that the ratio is in agreement with that of Mohn and Beckmann (2002). The magnitudes of the currents as such are much larger in Mohn and Beckmann (2002), due to the fact that their measurements were made over the top of the seamount, where water depth is smaller (by about a factor of five). They also found that the diurnal components are strongly reduced off the seamount; in the neighbouring open ocean, they form a much smaller fraction (order one-tenth) of the total tidal signal.

The results for the baroclinic cross-slope component, $u^{\prime}$, are shown in Fig. 7a, d. The semi-diurnal constituent (red line) has its largest amplitudes in the upper $500 \mathrm{~m}$ of the water column, and is generally stronger than the diurnal constituent, except near $300 \mathrm{~m}$ depth, where the latter peaks (blue). The semi-diurnal phase shows a clear upward increase between 300-600 $\mathrm{m}$ depth, indicating upward phase propagation and hence downward energy propagation. The phases are here represented in "unwrapped" angles; as a consequence, they cover intervals larger than the strictly necessary length of $2 \pi$. (This is done for clarity of presentation; otherwise the diurnal constituents, in particular, would give rise to highly erratic plots, due to the jumps from 0 to $2 \pi$, and vice versa, which of course have no physical significance in themselves.)

The remaining panels of Fig. 7 show amplitudes and phases of the along-slope baroclinic current velocity $v^{\prime}$, and of buoyancy $b$. (The latter represents the total, i.e. barotropic 
plus baroclinic signal; we determine its baroclinic part in Sect. 4.2.) Overall, the phase of the semi-diurnal constituent of $v^{\prime}$ lags that of $u^{\prime}$ by values of around $\pi / 2$ (typically between 1.3 and 1.8 in the upper $600 \mathrm{~m}$ ), consistent with the idea of along-slope uniformity (which we assume in Sect. 5), which implies $v_{t}^{\prime}=-f u^{\prime}$ and hence gives rise to a phase shift of $\pi / 2$. The diurnal across and along-slope components both show a distinct peak at around $300 \mathrm{~m}$ depth, with nearly identical amplitudes, consistent with circular polarization, as may be expected at this near-inertial frequency. The numerical experiments, discussed in Sect. 5, suggest that the peak is not of tidal origin.

The harmonic constituents, taken together, give a reasonably faithful description of the original signal. The superposition of the semi-diurnal, diurnal, and (the overall weak) quarter-diurnal constituents deviates on average (in time and vertically) from the original signal by $0.012 \mathrm{~m} \mathrm{~s}^{-1}$ for the cross-slope baroclinic component (rms-value: $0.043 \mathrm{~m} \mathrm{~s}^{-1}$ ), by $0.013 \mathrm{~m} \mathrm{~s}^{-1}$ for the along-slope baroclinic component (rms-value: $0.038 \mathrm{~m} \mathrm{~s}^{-1}$ ), and by $6.4 \times 10^{-5} \mathrm{~m} \mathrm{~s}^{-2}$ for buoyancy (rms-value: $2.0 \times 10^{-4} \mathrm{~m} \mathrm{~s}^{-2}$ ).

\section{Energy fluxes}

The basic definition of internal-tide energy flux reads

$E_{f}=\left\langle u^{\prime} p^{\prime}\right\rangle$,

where the baroclinic velocity $u^{\prime}$ is calculated from observed profiles by subtracting the depth-averaged part (which is presumed to represent the barotropic signal). The principal difficulty lies in finding the baroclinic pressure, $p^{\prime}$; we discuss this problem first.

\subsection{Indeterminacy in energy-flux profiles}

We start with the linear hydrostatic momentum equations

$$
\begin{aligned}
u_{t}-f v & =-p_{x} \\
v_{t}+f u & =-p_{y} \\
p_{z} & =b
\end{aligned}
$$

where $p$ is pressure (now divided by a constant reference value of density, $\rho_{*}$ ), and $b$ buoyancy, defined in Eq. (1). These quantities represent the barotropic plus baroclinic fields; in Eq. (6), the static fields have been left out. We note that because $p$ is here defined as pressure divided by $\rho_{*}$, the definition of energy-flux (Eq. 3) changes into $E_{f}=\rho_{*}\left\langle u^{\prime} p^{\prime}\right\rangle$.

To calculate the internal-tide energy flux, we need to distill first their baroclinic parts (denoted by primes). For the horizontal velocity components, we do so by subtracting the depth-average values:

$u^{\prime}=u-\frac{1}{h} \int_{-h}^{0} d z u ; \quad v^{\prime}=v-\frac{1}{h} \int_{-h}^{0} d z v$.
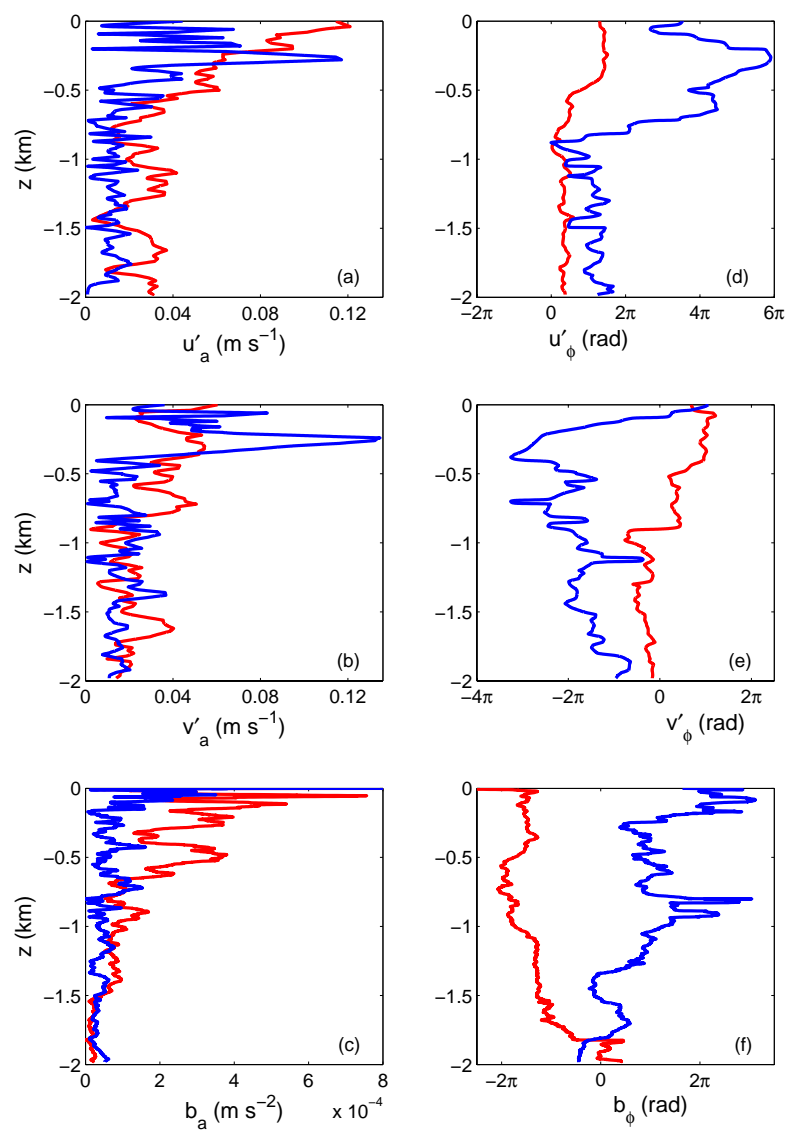

Fig. 7. The semi-diurnal and diurnal constituents of the crossand along-slope baroclinic velocity ( $u^{\prime}$ and $v^{\prime}$, respectively), and of buoyancy $b$. Left panels show the amplitudes; right panels, the phases. In each panel, the semi-diurnal (red line) and diurnal (blue) constituents are shown.

Here the surface is placed at $z=0$, and the bottom at $z=-h(x, y)$; we do not assume uniform depth. By construction, the vertical integrals of $u^{\prime}$ and $v^{\prime}$ are zero, a property we may refer to as the "baroclinicity condition for velocity".

The other baroclinic quantity we need is pressure $p^{\prime}$, which is related to $b^{\prime}$ via the hydrostatic balance, $p_{z}^{\prime}=b^{\prime}$. For the moment we shall suppose we have been able to determine $b^{\prime}$ (we return to this point in Sect. 4.2), and focus henceforth on deriving $p^{\prime}$ from it.

The hydrostatic balance implies

$p^{\prime}(t, x, y, z)=p^{\prime}\left(t, x, y, z_{0}\right)+\int_{z_{0}}^{z} d \bar{z} b^{\prime}(t, x, y, \bar{z})$,

where the first term on the right is a "constant" of integration; the value of $z_{0}$ is arbitrary, but natural choices are $z_{0}=0$ (surface) or $z_{0}=-h(x, y)$ (bottom).

Garcia Lafuente et al. (1999) took the former, but neglected, without any justification, the constant of integration. This amounts to assuming that baroclinic pressure vanishes 


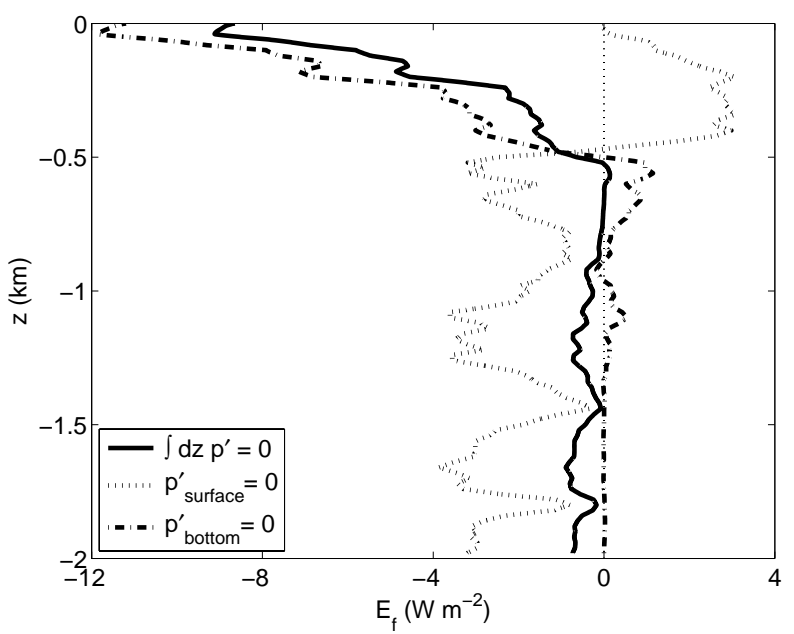

Fig. 8. Energy-flux profiles for the semi-diurnal internal tide, based on different ways of evaluating baroclinic pressure: the solid line is based on the assumption that the vertical integral of baroclinic pressure is zero ("Kunze condition"); the dotted line assumes baroclinic pressure to be zero at the surface ("Garcia Lafuente condition"); the dash-dotted line assumes it to be zero at the bottom.

at the surface, an assumption rightly criticized by Kunze et al. (2002). (We note that baroclinic surface pressure does not even vanish under the rigid-lid approximation - assuming it does is an elementary misconception that occasionally surfaces in the literature.)

The central problem - to determine the constant of integration - thus remains. To solve this, Kunze et al. (2002) proposed a "baroclinicity condition for pressure", meaning that the vertically integrated baroclinic pressure must be zero; this would indeed fix the constant. However, this condition is incompatible with the other baroclinicity condition, that for velocity - except in the absence of topography (i.e. if the bottom is purely horizontal). This point seems to have passed unnoticed in the literature, but it is easy to prove. To begin with, it is clear from Eqs. (4) and (5), applied to the baroclinic fields, that the baroclinicity condition for velocity implies

$\int_{-h}^{0} d z p_{x}^{\prime}=0 ; \quad \int_{-h}^{0} d z p_{y}^{\prime}=0$.

Thus, the vertically integrated horizontal derivatives of baroclinic pressure vanish. Moreover, we have the mathematical identity

$\frac{\partial}{\partial x} \int_{-h(x, y)}^{0} d z p^{\prime}=\left.p^{\prime}\right|_{z=-h} h_{x}+\int_{-h(x, y)}^{0} d z p_{x}^{\prime}$

(and an analogous expression in terms of the $y$ derivative). The second term on the right is zero because of Eq. (9). The first term on the right, however, contains the baroclinic pressure at the bottom, which in general is not zero. It thus follows that, in the presence of topography, the vertically inte- grated baroclinic pressure cannot be assumed to be zero. In fact, even if the baroclinic bottom pressure were assumed to be zero, it may still be inconsistent to require the vertically integrated pressure to be zero, because this requirement may yield a profile in which the value at the bottom is nonzero, contradicting the original assumption. (The profile in Fig. 8, solid line, is a case in point.) The failure of the "baroclinicity condition for pressure", which was meant to fix the constant of integration in Eq. (8), means that we are left with an indeterminacy in the energy-flux profiles.

Note that energy-flux profiles in the $y$ direction too suffer from an indeterminacy even if $\partial h / \partial y=0$. The presence of a slope in $x(\partial h / \partial x \neq 0)$ is sufficient to invalidate the "baroclinity condition for pressure"; and the resulting failure to fix the constant of integration automatically has a bearing on the $y$ direction as well; after all, the same (undetermined) constant of integration is at stake in $\left\langle v^{\prime} p^{\prime}\right\rangle$.

In the absence of any topography, on the other hand, we can write the baroclinic vertical velocity as a sum of modes $W_{n}(z) \exp i\left(k_{n} x+l_{n} y-\sigma t\right)$ (summing over mode number $n$ ), in which case the baroclinic pressure and horizontal velocities are all proportional to its vertical derivative $d W_{n} / d z$; it then follows immediately that the vertical integrals of these quantities must be zero (since $W$ vanishes at the surface and bottom).

The underlying cause why the presence of a slope spoils the "baroclinicity condition for pressure" proposed by Kunze et al. (2002), lies in the non-separable nature of the problem. In the absence of topography, separation of horizontal and vertical coordinates applies, and one can deal with the vertical structure independently of the horizontal position. In the presence of topography, the two become intertwined. Indeed, it is clear from Eq. (8) that one could find the "constant" of integration, which is due to vertical integration, from information of the horizontal dependence of velocity. (Specifically, taking $z_{0}=0$, one could find the constant by horizontally integrating Eqs. (4) and (5), with respect to $x$ and $y$, respectively.) But from measurements at a single station, such information is simply not available.

As the problem seems to be fundamentally unsolvable, this leaves us no other choice than a pragmatic approach. As a matter of fact, in its source region, i.e. over the slope, the internal tide is usually concentrated in a beam. Suppose, for example, that the beam is located in the upper layer of the water column, and that baroclinic currents are very weak in the lower layer; then it makes sense to assume that all baroclinic fields, including pressure, are weak there. One may then simply assume the baroclinic pressure at the bottom to be zero.

To see how the choice of the level of zero pressure affects the energy-flux profiles, we consider three cases, all for the semi-diurnal internal tide (Fig. 8). (At this stage we ignore the barotropic contribution in $b$, and simply assume the observed $b$ to be entirely baroclinic, i.e., $b^{\prime}=b$; we return to this point below.) The solid line is based on the assumption of 
zero-integrated pressure as proposed by Kunze et al. (2002). Assuming baroclinic pressure to be zero at the bottom gives a somewhat different curve (dash-dotted line). Both show a clear negative flux in the upper $500 \mathrm{~m}$, i.e. directed away from the seamount, as one would expect because internal tides are generated near the top of the seamount, and, according to Fig. 7a (red line), the semi-diurnal cross-slope signal is particularly strong in the upper $500 \mathrm{~m}$. It is for this reason that the dotted line in Fig. 8 should be rejected as unphysical; it is based on the assumption of zero surface pressure.

We emphasize that the constant of integration affects only the energy-flux profiles, not their vertically integrated values, since the first term on the right-hand side of Eq. (8) plays no role in the vertically integrated $u^{\prime} p^{\prime}$, by virtue of the baroclinicity condition for velocity. So, for each of the three profiles in Fig. 8, the integrated value is the same, namely $-2.4 \mathrm{~kW} \mathrm{~m}^{-1}$.

\subsection{Results}

The buoyancy field shown in Figs. 3 and 7c, f contains a baroclinic as well as a barotropic tidal signal; the latter (which we denote by $B$ ) represents merely the movement of the isopycnals that is kinematically induced by the barotropic tidal flow over the slope. To calculate the baroclinic energy flux properly, this barotropic part should be removed. It can however not be directly deduced from the data, and some additional assumptions are needed. We assume that the barotropic cross-slope transport is spatially uniform; hence, for each tidal constituent, the cross-slope barotropic velocity can be written as $U=Q \sin (\sigma t-\Phi) / h(x)$, where $Q$ is the amplitude of the barotropic cross-slope flux. By continuity, the vertical barotropic component then becomes

$$
W=\frac{z Q \sin (\sigma t-\Phi)}{h(x)^{2}} h_{x}
$$

The barotropic part of buoyancy is then given by $B_{t}=-N^{2} W$. At the measurement site, $d h / d x \approx 0.14$. The remaining parameters $(Q, \Phi)$ follow from the harmonic analysis. This allows to remove the barotropic part $B$ from $b$. The correction thus made, however, is small; for example, for the semi-diurnal component the difference between the amplitudes of $b$ and $b^{\prime}=b-B$ is, on average, only $4 \times 10^{-5} \mathrm{~m} \mathrm{~s}^{-2}$ (cf. Fig. 7c, red line).

Next we integrate $b^{\prime}$ vertically to obtain baroclinic pressure, following Eq. (8), and then, by the procedure described in the previous section, the vertically integrated energy flux. The results are: $-2.3 \mathrm{~kW} \mathrm{~m}^{-1}$ (semi-diurnal) and $+0.12 \mathrm{~kW} \mathrm{~m}^{-1}$ (diurnal); negative (positive) means a net flux away from (toward) the seamount. The magnitude of the semi-diurnal flux is slightly smaller than the value given at the end of the previous section; this is because we have here properly calculated $b^{\prime}=b-B$, whereas the earlier value was simply based on the assumption that $B$ is negligible. To shed more light on the energy fluxes of the semi-diurnal and di- urnal components, we now consider results from numerical experiments.

\section{Numerical modelling}

We compare the energy fluxes obtained from the yoyo measurements with those from a linear hydrostatic internal-tide model that was previously used to estimate energy fluxes in the Bay of Biscay (Gerkema et al., 2004); the model assumes uniformity in the along-slope direction. The required input consists of three things: a vertical profile of buoyancy frequency $N$, for which we use Fig. 2c; a topographic profile, for which we use the track shown in Fig. 1; and the crossslope barotropic tidal transports $(Q)$. The latter can be derived from the barotropic current amplitudes mentioned in Sect. 3 (see also Fig. 6), by multiplying with the local water depth $(1980 \mathrm{~m})$; this gives $Q=39.6$ (semi-diurnal) and 14.9 (diurnal), both in $\mathrm{m}^{2} \mathrm{~s}^{-1}$. The resulting pattern for the semidiurnal tide, in terms of the amplitude of baroclinic $u^{\prime}$, is shown in Fig. 9. The lower panel shows the corresponding amplitude profile of $u^{\prime}$ at the location of the yoyo-station; this profile is compared with the observed one (dotted line). In both, the largest amplitudes occur in the upper $200 \mathrm{~m}$, but the observed signal has a much smaller amplitude and is much wider, in other words, it is more smeared out than the beam in the numerical model. These effects of amplitude reduction and widening partly compensate each other in a depthintegrated sense. This becomes apparent if one calculates the vertically integrated energy flux, which is $-2.6 \mathrm{~kW} \mathrm{~m}^{-1}$, being only $13 \%$ larger in magnitude than the observed value (which was $-2.3 \mathrm{~kW} \mathrm{~m}^{-1}$ ).

For the diurnal component, the signal is much weaker (Fig. 10), since the cross-slope barotropic component, which determines the forcing, is about 2.6 times weaker. The energy flux is here predominantly negative: the model yields a vertically integrated energy flux of $-0.034 \mathrm{~kW} \mathrm{~m}^{-1}$, consistent with the idea of internal-tide propagation away from the seamount. Recall that the observed value was positive, and moreover much larger: $+0.12 \mathrm{~kW} \mathrm{~m}^{-1}$. Part of the explanation may lie in the fact that in the observed results, nearinertial internal waves dominate the "diurnal" signal that are not due to barotropic tidal forcing and hence not reproduced by the model.

Barotropic to baroclinic conversion is only one of the potential mechanisms for the generation of diurnal signals at this location. Another mechanism is subharmonic resonance (e.g., Hibiya et al., 2002; MacKinnon and Winters, 2005; Gerkema et al., 2006): semi-diurnal internal tides may by parametric subharmonic instability excite internal tides of half that frequency at latitudes where the latter can exist as a free wave (i.e. equatorward of $29.9^{\circ} \mathrm{S} / \mathrm{N}$ for $\mathrm{S}_{2}$, and $28.8^{\circ} \mathrm{S} / \mathrm{N}$ for $\mathrm{M}_{2}$ ). For $\mathrm{S}_{2}$ this process may occur at the southern flank, but for $\mathrm{M}_{2}$ only at some southward distance from Great Meteor Seamount. (We note that in defining the 

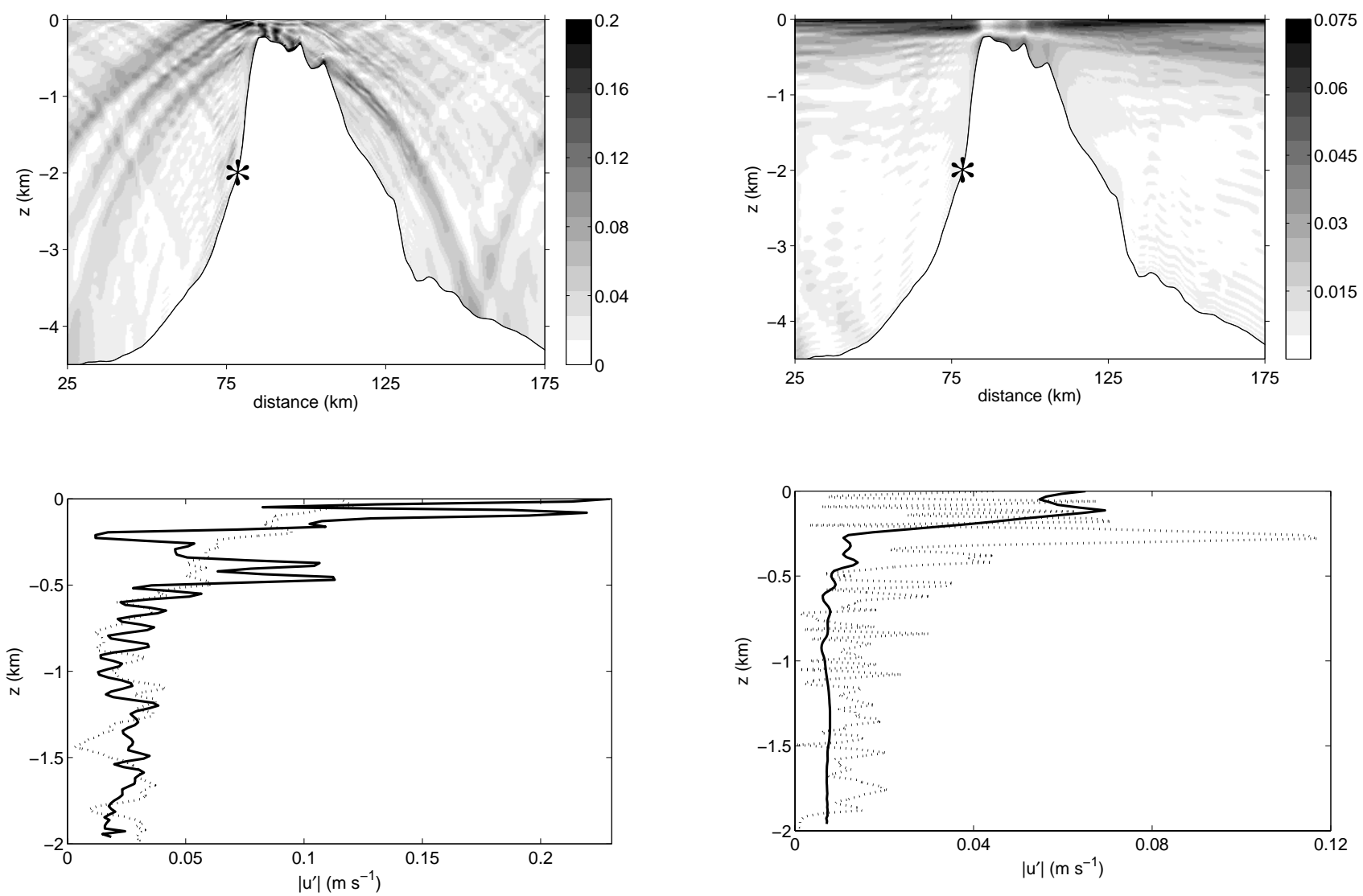

Fig. 9. The numerically modelled amplitude of the baroclinic semidiurnal cross-slope current, $\left|u^{\prime}\right|$ (in $\mathrm{m} \mathrm{s}^{-1}$ ). Below, the corresponding modelled profile (solid line) at the yoyo position (marked by an asterisk above); in the same panel, the observed profile is shown (dotted line), reproduced from Fig. 7a.

"critical" latitude, we use the "traditional" definition according to which it is the latitude where the tidal frequency equals the local Coriolis parameter $f$; in weakly stratified regions, such as the abyssal ocean, this definition requires modification, as pointed out by Gerkema and Shrira (2005).) These " $\mathrm{S}_{1}$ " and " $\mathrm{M}_{1}$ " diurnal frequencies moreover lie close to the local inertial frequency $f$ (which at this latitude shows an enhanced spectral peak, see van Haren, 2005b), at which nearinertial waves occur due to atmospheric forcing, a third possible source of the "diurnal" energy found in the measurements.

To return to the semi-diurnal tidal energy flux, the measurements made here at a single location do not allow us to infer with any certainty how much Great Meteor Seamount as a whole contributes to the barotropic/baroclinic energy conversion. Still, to get an idea of the order of magnitude, we extrapolate the value found here to the entire seamount, multiplying $2.3 \mathrm{~kW} \mathrm{~m}^{-1}$ by the circumference of a circle, the radius of which is (roughly) estimated to be $20 \mathrm{~km}$. This gives

a total conversion of $0.3 \mathrm{GW}$, which is about sixty times less than at the Hawaiian Ridge (Klymak et al., 2006).

\section{Conclusions}

In estimating energy fluxes over Great Meteor Seamount, we have focussed on vertically integrated values rather than vertical profiles, because, as argued in Sect. 4.1, the latter are fundamentally ambiguous over topographic features - a point not previously noted in the literature. Over a sloping bottom the "baroclinicity condition for pressure", as proposed by Kunze et al. (2002), fails to be valid. This failure is frustrating, since the primary interest of internal-tide energy fluxes lies in regions of strong topography! Fortunately, the vertically integrated values can be determined unambiguously.

We found that the observed semi-diurnal internal-tide energy flux is very similar to the one found from a numerical model; also the location of large amplitudes is correctly modelled, but the model represents the internal tide as a more 
intense, peaked beam than is found in the observations. The differences between model and observations are much larger for the diurnal signal, which at this latitude coincides with near-inertial signal. The observations yield a northward energy flux, i.e. towards the seamount, which is not only directionally opposed to the model result, but also much larger in amplitude. This is plausibly due to the fact that the mechanisms behind near-inertial waves (primarily the wind) are not included in the model. Still another mechanism may be responsible for the enhanced diurnal/inertial signal, namely parametric instability of the $S_{2}$ tide, creating a subharmonic (which is not included in the model, either).

The semi-diurnal internal-tide energy flux, according to model and observations, is smaller than found for example in the Bay of Biscay, but only by a factor of four. The reason that the flux is not much smaller is that the plateau of Great Meteor Seamount, although obviously deeper than the shelf in the Bay of Biscay, still lies shallow enough for the slope to cross the permanent pycnocline, which was earlier shown to be a major factor in internal-tide generation (Gerkema et al., 2004).

Acknowledgements. We gratefully acknowledge the help of the crew on R/V Pelagia; the data was made ready for use by M. Hiehle (CTD) and C. Veth (LADCP). The cruise during which these measurements were made forms part of the LOCO-project (NWO-groot) and BSIK. We thank E. Kunze for comments on an earlier version of this paper, and apologize for the "fiendishly clever" refutation of his condition. T. Gerkema receives funding from the NWO/ALW project CLIMA-DIM.

Edited by: J. M. Huthnance

\section{References}

Dietrich, G.: Ozeanographie - physische Geographie des Weltmeeres, Georg Westermann Verlag, Braunschweig, 1970.

Egbert, G. D. and Ray, R. D.: Semi-diurnal and diurnal tidal dissipation from TOPEX/Poseidon altimetry, Geophys. Res. Lett., 30, 1907, doi:10.1029/2003GL017676, 2003.

Feistel, R. and Hagen, E.: On the GIBBS thermodynamic potential of seawater, Progr. Oceanogr., 36, 249-327, 1995.

Garcia Lafuente, J., Sarhan, T., Vargas, M., Vargas J. M., and Plaza, F.: Tidal motions and tidally-induced fluxes through La Linea submarine canyon, western Alboran Sea, J. Geophys. Res., 94, 18 185-18 194, 1999.
Gerkema, T., Lam, F. P. A., and Maas, L. R. M.: Internal tides in the Bay of Biscay: conversion rates and seasonal effects, Deep-Sea Res. II, 51, 2995-3008, 2004.

Gerkema, T. and Shrira, V. I.: Near-inertial waves on the "non-traditional" $\beta$ plane, J. Geophys. Res., 110, C01003, doi:10.1029/2004JC002519, 2005.

Gerkema, T., Staquet, C., and Bouruet-Aubertot, P.: Decay of semidiurnal internal-tide beams due to subharmonic resonance, Geophys. Res. Lett., 33, L08604, doi:10.1029/2005GL025105, 2006.

Hibiya, T., Nagasawa, M., and Niwa, Y.: Nonlinear energy transfer within the oceanic internal wave spectrum at mid and high latitudes, J. Geophys. Res., 107(C11), 3207, doi:10.1029/2001JC001210, 2002.

Klymak, J. M., Moum, J. N., Nash, J. D., Kunze, E., Girton, J. B., Carter, G. S., Lee, C. M., Sanford, T. B., and Gregg, M. C.: An estimate of tidal energy lost to turbulence at the Hawaiian Ridge, J. Phys. Oceanogr., 36, 1148-1164, 2006.

Kunze, E., Rosenfeld, L. K., Carter, G. S., and Gregg, M. C.: Internal waves in Monterey Submarine Canyon, J. Phys. Oceanogr., 32, 1890-1913, 2002.

MacKinnon, J. A. and Winters, K. B.: Subtropical catastrophe: Significant loss of low-mode tidal energy at $28.9^{\circ} \mathrm{N}$, Geophys. Res. Lett., 32, L15605, doi:10.1029/2005GL023376, 2005.

Mohn, C. and Beckmann, A.: The upper ocean circulation at Great Meteor Seamount. Part I: Structure of density and flow fields, Ocean Dynamics, 52, 179-193, doi:10.1007/s10236-002-00174, 2002.

Nash, J. D., Alford, M. H., and Kunze, E.: Estimating internal wave energy fluxes in the ocean, J. Atmos. Oceanic Techn., 22, 15511570, 2005.

Nash, J. D., Kunze, E., Lee, C. M., and Sanford, T. B.: Structure of the baroclinic tide generated at Kaena Ridge, Hawaii, J. Phys. Oceanogr., 36, 1123-1135, 2006.

Rainville, L. and Pinkel, R.: Baroclinic energy flux at the Hawaiian Ridge: observations from the R/P FLIP, J. Phys. Oceanogr., 36, 1104-1122, 2006.

Smith, W. H. F. and Sandwell, D. T.: Global sea floor topography from satellite altimetry and ship depth soundings, Science, 277, 1956-1962, 1997.

van Haren, H.: Details of stratification in a sloping bottom boundary layer of Great Meteor Seamount, Geophys. Res. Lett., 32, L07606, doi:10.1029/2004GL022298, 2005a.

van Haren, H.: Tidal and near-inertial peak variations around the diurnal critical latitude, Geophys. Res. Lett., 32, L23611, doi:10.1029/2005GL024160, 2005b.

van Haren, H.: Cruise report bsik/LOCO-IW06: R.V. Pelagia cruise 64PE248, 17 May-12 June 2006, NIOZ, 2006.

Zaron, E. D. and Egbert, G. D.: Estimating open-ocean barotropic tidal dissipation: the Hawaiian Ridge, J. Phys. Oceanogr., 36, 1019-1035, 2006. 